\title{
Group Risk-Taking Under Various Group Decision Schemes
}

\author{
Robert B. Zajonc, Robert J. Wolosin, and Mrrna A. Wolosin \\ The University of Michigan and Indiana University
}

\begin{abstract}
This study examined individual choice behavior when it occurs in groups engaged in joint decision-making. Ninety-six subjects made binary choices between "risky" and "conservative" alternatives acting alone and acting as members of three-man groups in a series of 360 trials. The groups operated under the majority rule, unanimity, and the so-called "minimal quorum" rule. It was found that under unanimity and the minimal quorum rule there were strong pressures toward uniformity. However, individual members in these groups did not succumb to these pressures by modifying their overall choice preferences. Instead they arranged their sequences of choices so that the joint choice of all members would be congruent with their acceptable level of risk-taking. The findings also indicated that there was a slight shift toward conservatism when individuals joined groups. The results on individual risk-taking supported a parameter-free model, and the model also accommodated the present group data when some new assumptions were made about the effects of pressures toward uniformity.
\end{abstract}

In studies of group decision-making it is often found that the group choice differs from the average of the individual members' choices. This is especially true of studies on decisions made under uncertainty which involve risk. Normally, in these studies, first observed are choices of single individuals, who are then formed into groups for the purpose of coming to a joint decision. The decisions of these groups often seem to be at variance with the average of individual decisions.

Two components of the difference betwecn individual and group choices were previously distinguished (Zajonc, Wolosin, Wolosin \& Sherman, 1968, 1969). First, it is possible that individual choice preferences change when people act in groups. In fact, theories which attempt to explain the so-called "risky shift" phenomenon limit themselves almost exclusively to this component factor and seek to isolate its causes. One theory holds that in groups, responsibility for the decision can be diffused among the individuals (Wallach, Kogan \& Bem, 1964); others hold that the group provides the individuals with knowledge about cultural values regarding the alternatives (Brown, 1965; Stoner, 1968);

(c) 1972 by Academic Press, Inc. 
or that he becomes more familiar with the decision problem (Bateson, 1966; Flanders \& Thistlethwaite, 1967); or that some members are more influential than others (Clausen, 1965; Wallach, Kogan \& Burt, 1968). Each of these theories assumes that if there is a change from individual to group decision-making, something happens to individual preferences for the choice alternatives when the choices are made jointly and in a group setting.

The second component factor of the difference between individual and group decisions, which is independent of the first, focuses on the ways in which individual choices are combined to form a joint decision. A difference between the average of individual choices and the joint decision can be obtained without any change in individual preferences (Zajonc, Wolosin, Wolosin \& Sherman, 1968). For instance, if there are two alternative courses of action, $A$ and $B$, and if, on the average, the individual members' preference of $\mathrm{A}$ is $70 \%$, then a group composed of these individuals and observing the majority rule will choose $A$ with a likelihood greater than 7 , without any of the members changing their choice preferences. But if the group operates under unanimity then the likelihood of such a group choosing $\mathrm{A}$ is much smaller than .7. Various group decision schemes were compared by Smoke and Zajonc (1962) in terms of their formal properties, and it was clear from the analysis that a variety of discrepancies between the average of individual choices and the joint decision can be obtained all with the individual choice preferences remaining constant. These discrepancies depend only on the group decision scheme.

In all studies on risky shift known to the authors (including those carried out by them) these two components of the discrepancy between individual and group choices are allowed to vary simultaneously and are confounded. In some experiments the group is instructed to reach "consensus" as, for instance, in the early study by Wallach and Kogan (1965), and in others such requirement is not made explicit, although the group is expected to come up with a single choice nevertheless. The method of arriving at "consensus" or the single choice is usually left to the group. One believes that these groups generally observe the majority rule, but we have no hard information to support this conjecture. Hence, if change does occur, it is impossible to determine whether it occurred because of changes in individual choice preferences, because of a particular decision scheme adopted, or because of an interplay between the two components.

The purpose of this study is to examine whether changes in individual choice preferences do occur in groups when group decision schemes are strictly controlled. If we had control over the decision scheme which 
combined the individual preferences of each member into a group decision it would be possible to separate the effects of pooling from the effects of group-produced changes in choice preferences. In the study reported here, individuals and groups are confronted with a two-choice situation where one event occurs twice as often as another and where a correct prediction of the rare event brings about a payoff that is twice as high as the payoff for a correct prediction of the frequent event. According to previous conventions, the prediction of the rare event constitutes a risky response while the prediction of the frequent event a conservative response. Groups which make decisions according to a majority rule are compared with groups which make decisions according to unanimity favoring risk (i.e., the infrequent event) or according to what has been previously called the "minimal quorum" (Smoke \& Zajonc, 1962). That is, in the first case the group as a whole makes a risky choice if more than half of its members make risky choices. In the second case the group as a whole makes a risky choice if and only if all members make risky choices, and a conservative choice otherwise. In the third case (minimal quorum) the group as a whole makes a risky choice if at least one member makes a risky choice. If no member chooses the rare event, the group decision favors the conservative alternative.

A second purpose of this study is to provide a further validation for a model of group decision-making under risk presented earlier (Zajonc, Wolosin, Wolosin \& Sherman, 1969). This parameter-free model was tested for two-choice situations with event probabilities of .6-.4 and .8-2. It is of interest to determine whether the model represents a viable approach to the problem of group decisions by examining it under a new set of event probabilities, namely .67-.33.

\section{METHOD}

\section{Subjects}

Ninety-six male undergraduates were recruited from the paid psychology subject pool at the University of Michigan. They were assigned randomly to four experimental groups. A minimum wage of $\$ 1.25 / \mathrm{hr}$ was guaranteed. When first contacted, subjects were told neither the purpose of the experiment nor that they could earn a greater sum of money during its course.

\section{Apparatus}

Two $1 / 25$ watt lights, mounted on a small panel, served to signal stimulus events. One was marked " 1 cent," and the other was marked " 2 cents." For half of the subjects, the left stimulus light was associated with the high payoff (and hence with the low probability of occurrence), and for the remainder, with the low payoff (and hence the high probability of occurrence). A punched tape program controlled the timing and sequence of stimulus events. Subjects were equipped with switches for making decisions and poker chips for recording their winnings. 


\section{Design and Procedure}

All subjects were given 360 trials in two consecutive sets of 180 each. They were treated in an identical way during the first 180 trials. The two stimuli were programmed with .67 and .33 probabilities of occurrence. The payoff for the highfrequency light was 1 cent, and for the low-frequency light, the payoff was 2 cents. The sequence of stimuli was random with the constraint that these probabilities be observed within each block of 30 trials.

Upon arriving at the laboratory, subjects were met by the experimenter and taken to individual cubicles where they worked alone for the first 180 trials. The following taped instructions were given to the subjects over an intercom:

In front of you, you see two lights. Every $7 \mathrm{sec}$, one or the other will come on. You also see two response plates. During each 7 -sec interval, you are to press one plate or the other to anticipate which light will come on. If you press the left plate and the left light comes on, you win 1 cent ( 2 cents). If you press the right plate and the right light comes on, you win 2 cents ( 1 cent). If you press one plate and the opposite light comes on, you win nothing. Also, if you fail to press within the 7 -sec interval you win nothing.

You can keep track of your winnings with the chips which you see in front of you. If you win 1 cent by anticipating the left (right) light, place a white chip into your bank. If you win 2 cents by anticipating the right (left) light, place a blue chip into your bank. Your winnings will also be kept automatically. You are guaranteed a minimum of $\$ 1.25 / \mathrm{hr}$ and you may keep whatever you win over that amount; that is, you will get either $\$ 1.25 / \mathrm{hr}$, or your winnings, whichever is more.

Stimuli were presented every $7 \mathrm{sec}$ and, unlike the previous experiments, there were no rest periods between trial blocks. Blue chips were always associated with the high payoff light, and white chips with the luw payoff light.

The second set of 180 trials differed among the 4 treatments of the experiment.

I-I Treatment. At the end of the 180 trials, subjects in the Individual-Individual (I-I) treatment were told that the first part of the experiment was over. They were then taken out of their cubicles by the experimenter and allowed to relax for about 3 or $4 \mathrm{~min}$. However, they were not permitted to speak to one another. After the rest period, they were taken back to their cubicles and they continued as previously for another set of 180 trials.

In the remaining treatments subjects worked in groups of three during the second set of 180 trials.

I-M Treatment. At the end of 180 trials, subjects in the Individual-Majority Vote (I-M) treatment were told that the first part of the experiment was over. The experimenter then met all three subjects and led them to a room equipped with apparatus identical to that which they had used alone. Taped instructions were again issued over the intercom:

Again you see the lights and the plates. Now you are working as a team that is making decisions as a group. This time, every $15 \mathrm{sec}$ one or the other light will appear. During this interval, each of you is to press one or the other of your plates indicating which light you have chosen, just as before. This time, however, you will also raise your hand so that everyone of you can see what the others have chosen. If you decide on the left light, press the left plate and raise your left hand. If you choose the right light, press the right plate and raise your right hand. 
I will now tell you how your individual decisions combine to make a group decision. The light which is chosen by at least two of you is your group decision. For example, if two of you have picked the left light and one has picked the right, then the group decision is for the left light. If two of you pick the right light and one has picked the left, your group decision is for the right light.

If your group decision is for left and the left light comes on, each of you wins 1 cent ( 2 cents). If your group decision is for the right, and the right light comes on, each of you wins 2 cents ( 1 cent). If your group decision is for one light and the other comes on, each of you wins nothing. Each man's pay during this group session depends on the group's decisions. None of you can win more than any other and either you all win or you all lose on every trial. Again you can keep track of your winnings by placing the appropriate chips into your bank. Are there any questions?

I-MQ Treatment. Subjects in the Individual-Minimal Quorum (I-MQ) treatment were told at the end of the first 180 trials that the first part of the experiment was over. After they were ushered into their group room by the experimenter, instructions similar to those from the I-M treatment were played, with the appropriate changes as follows:

Again you see the lights and the plates. Now you are working as a team ... I will now tell you how your individual decisions combine to make a group decision. If all three of you choose the left light, then the left light is your group decision. If at least one of you chooses the right light, then your group decision is for right. In other words, the only way for your group decision to be for the left light is if all three of you choose left.

If your group decision is for left, and the left light comes on, each of you wins 1 cent. . .

In this treatment, the group choice was for the risky alternative when any one of the three group members made such a vote. A conservative group choice could occur therefore only when all members voted the conservative alternative.

$I-U$ Treatment. In the Individual-Unanimity treatment (I-U), subjects were again placed into the group room after their initial 180 trials. These subjects heard the standard instructions. The instructions were modified to communicate that the group could make a risky choice if and only if all three members voted for the infrequent alternative. ${ }^{1}$

In each of the above group conditions 180 trials separated by 15 -sec intervals were given. As previously, the probabilities were $2 / 3$ and $1 / 3$ and they were observed for each hlock of 30 trials.

The subjects were not permitted to discuss their choices, but could talk about matters irrelevant to the task. Also, they were required to vote at the same time. Monitoring of the discussion over an intercom showed that the above constraints prevailed.

${ }^{1}$ Precisely, the difference between I-U and I-MQ is this: If A designates the highfrequency light and $\mathrm{B}$ the low-frequency light, then there are 1 combinations of 3 individual choices: $A A A, A A B, A B B$, and BBB. In $I-U$, a group with the combination $B B B$ would be the only one to vote for $B$, while groups with any of the other three combinations would be voting for A. In I-MQ, all combinations except AAA would result in a vote for $B$. 
After the experimental session, an explanation of the experiment was given and subjects were paid their wages.

\section{RESUITS}

\section{Individual and Group Choices}

Results are reported in percentages of risky choices. Table 1 shows these choices for the control (I-I) and the three experimental (I-U, I-MQ, and I-M) conditions for both sessions of the experiment. In the experimental conditions, scores during the first session were the average percentage of risky choices made by the group members individually, and in the second session, they were the percentage of group decisions for the risky alternative. As in our earlier studies, data from the control condition were aggregated into random sets of three-man statisticized "groups"; responses of these "groups" were averaged for both sets of 180 trials.

An analysis of variance was carried out with treatments as the between-subjects factor and trial blocks (with the sessions nested within blocks) as the within-subject factor. For the purposes of this analysis degrees of freedom in the error term were reduced because of the grouping of individual scores in the second session of the I-I condition. The analysis showed significant overall effects of trial blocks $(F(11,308)=8.00, \quad p<.001)$ and sessions $(F(1,308)=52.50, \quad p<$ $.001)$. There was also a significant interaction between treatments and sessions $(F(3,308)=4.38, p<.001)$. All groups showed declines in risktaking (i.e., shifts in the conservative direction). The smallest decline occurred in the I-MQ treatment, while the largest decline was shown by I-U groups.

Because of the significant interaction between sessions and treatments, simple effects tests (as outlined by Winer, 1962, pp. 310-311) were

TABLE 1

Percentage of Risky Choichs Made by Individuan and Groups

\begin{tabular}{lcccc} 
& $\begin{array}{c}\text { First } 180 \\
\text { trials }\end{array}$ & \multicolumn{2}{c}{$\begin{array}{c}\text { Second } 180 \\
\text { trials }\end{array}$} \\
\cline { 2 - 3 } Treatment & $\begin{array}{c}\text { Individual } \\
\text { choices }\end{array}$ & $\begin{array}{c}\text { Individual } \\
\text { choices }\end{array}$ & $\begin{array}{c}\text { Group } \\
\text { choices }\end{array}$ \\
\hline I-I (Individual-Individual) & 46.1 & 37.7 & 37.7 \\
I-M (Individual-Majority) & 44.7 & 41.4 & 39.0 \\
I-U (Individual-Unanimity) & 47.6 & 42.2 & 34.7 \\
I-MQ (Individual-Minimal Quorum) & 45.2 & 33.2 & 42.4 \\
\hline
\end{tabular}


carried out. These tests showed that choices in the first session differed significantly from choices in the second session (at the .01 level or better) in the I-M and I-U treatments, but also in the control (I-I) treatment." Only in the I-MQ treatment was the decline in risk-taking nonsignificant. Furthermore, the 4 treatments did not differ among themselves for the first 180 trials $(F(3,21)<1.00)$; the differences among them in the second session are only marginally significant $(F(3,21)=2.56, .10>$ $p>.05)$.

Table 1 also shows percentages of risky choices made by individuals when in groups. These data were analyzed by analysis of variance similar to the one described above and including the first 180 trials, except that the error term maintained its full number of degrees of freedom. As before, significant effects for trial blocks $(F(11,1012)=10.62$, $p<.001)$ and sessions $(F(1,1012)=83.24, p<.001)$ occurred, and there was a significant interaction between sessions and treatments $(F(3,1012)=5.41, p<.001)$.

Simple effects tests were carried out. They indicate that while individual choices in the first session did not vary significantly as a function of treatment $(F(3,92)<1.00)$, they did vary significantly in the second half of the experiment $(F(3,92)=6.68, p<.01)$. We note from Table 1 that within experimental treatments, substantial decreases in average risktaking occurred. These changes were greatest in the I-MQ treatment $(F(1,1012)=28.54, p<.001)$, somewhat smaller but still significant in the I-U treatment $(F(1,1012)=5.49, p<.05)$, and least of all in the $\mathrm{I}-\mathrm{M}$ treatment $(F(1,1012)=2.28$, ns $)$. On the other hand, there was a significant decline in risk in the control (I-I) treatment $(F(1,1012)=13.75, p<.001)$.

\section{Group Decision Schemes}

It will be of interest to examine the present data in the light of the decision model presented in the earlier publication (Zajonc et al., 1969). This parameter-free model assumes that, given two alternative events, $\mathrm{A}$ and $\mathrm{B}$, occurring with the probabilities $p(\mathrm{~A})$ and $p(\mathrm{~B})$ with $p(\mathrm{~A})>$ $p(\mathrm{~B})$, the individual can find himself at the onset of any trial in any of three mutually exclusive states: (a) he is certain that A will occur and equally certain that B will not occur; (b) he is certain that B will occur

\footnotetext{
${ }^{2}$ The reason why there is a significant difference between the first and second 180 trials even in the I-I condition is that during the early trials individual choices hover around $50 \%$ before reaching an asymptote which is roughly the one that is maintained during the second 180 trials. Total rather than asymptotic performance was observed during the first 180 trials so that the data from the first and the second 180 trials can be based on the same number of observations.
} 
and equally certain that A will not occur; (c) he is uncertain which of the two events will occur. These three states can be simply called A, B, and $A \cup B$. An individual's probability of being in a given state is assumed to correspond to the objective probability of the given event. Thus, for example, his probability of being in state $A, P(A)$, is equal to the probability that $A$ will occur times the probability that $B$ will not occur; his probability of being in state $B, P(B)$, is the converse; and his probability of being in state $A \cup B, P(A \cup B)$, is the remainder. For the $.67-.33$ event probabilities used in the present experiment $\mathrm{P}(\mathrm{A})=$ $p(\mathrm{~A})[(1-p(\mathrm{~B})]=.44 ; \mathrm{P}(\mathrm{B})=p(\mathrm{~B})[(1-p(\mathrm{~A})]=.11$; and $\mathrm{P}(\mathrm{A} \cup$ $\mathrm{B})=1-\mathrm{P}(\mathrm{A})+\mathrm{P}(\mathrm{B})=.45$.

As in previous experiments, in the present one the subjects are required to predict either $\mathrm{A}$ or $\mathrm{B}$ and they cannot declare uncertainty. Hence, states $A$ and $B$ are stable, and when in these states the individual will make the response " $A$ " or " $B$," respectively, before the trial terminates. But $\mathrm{A} \cup \mathrm{B}$ is unstable, and the rules of the experiment require him to move from $A \cup B$ to another of two states, $A$ or $B$. It is thus assumed that the individual who is in state $\mathrm{A} \cup \mathrm{B}$ at the onset of the trial will move to $\mathrm{A}$ or to $\mathrm{B}$. For reasons discussed in the previous publication, the probabilities of moving to $A$ or to $B$ from $A \cup B$ are not proportional to the probabilities associated with the events $A$ or $B$, but they are proportional to the payoffs associated with these events. Hence, in the present case the individual who is in $\mathrm{A} \cup \mathrm{B}$ will move twice as often to $B$ than to $A$ because the payoff for $B$ is 2 cents and for $A$ only 1 cent. Table 2 shows the initial and terminal probabilities for the various states in the present experiment.

It can be seen from the above table that the model predicts $40.7 \%$ risky (i.e., B) choices when they are made individually. Actually, in the last 90 trials of the first session, which represent individual asymptotic

TABIL 2

Initial and Teminal Promabilties of Individua lisky Choicles Acconding to the Two-Sragh Mophe

Individual state on a given trial Initial stage Terminal stage

$\mathrm{A} \cup \mathrm{B}$
$\mathrm{B}$$\quad .445 \stackrel{1.0}{\longrightarrow} .39 \mathrm{C}$


performance, there were $41.7,44.9,44.5$, and $42.9 \%$ risky choices for the I-M, I-U, I-MQ, and I-I treatments, respectively. These values average to $43.5 \%$ risky choices, a figure not too far removed from that predicted by the model.

According to the decision rules required in the I-MQ and I-U treatments the model would predict $1-.593^{3}=.792$ risky choices in the $\mathrm{I}-\mathrm{MQ}$ treatment and $.407^{3}=.067$ risky choices in the I-U treatment. For the I-M treatment we would have to predict that the group makes a risky choice when either all three group members are in state B (which is equal to .067) or when two of them are in state B and one in state A (which is equal to .296). Hence, the probability that the group will choose the risky alternative when operating under the majority rule is predicted to be .363 .

This last figure is again quite close to the one obtained in the I-M treatment, namely .39 (see Table 1). A conservative shift is predicted by the model when the group operates by the majority rule, and such a shift is obtained. The predictions for the I-MQ and I-U treatments, however, are ntterly incorrect. The predicted probability of risky choices of .792 overestimates the I-MQ performance by nearly forty points, and the predicted probability of .067 risky choices underestimates the obtained performance of I-U groups by nearly thirty points.

How can these discrepancies be explained? Two possibilities exist. First, we can assume that individual choice preferences remain stable when subjects move from the alone condition to groups operating under the majority rule. But they change dramatically when individuals move to groups operating under unanimity or quorum. This conjecture, however, is contradicted by the evidence. It is seen in Table 1 that there is indeed a drop in risky choices for individuals going into I-MQ groups. But the change is negligible for individuals joining I-U groups. Moreover, the drop in individual risk-taking in the I-MQ treatment is not sufficient to explain the obtained results in this treatment. Since $33.2 \%$ of individual choices are risky choices (see Table 1), then we would expect in the $\mathrm{I}-\mathrm{MQ}$ condition $\mathrm{I}-(1-.332)^{3}=.702$ such group choices. This figure is somewhat smaller than the .792 predicted by the initial probabilities but still quite out of the range of the data.

The answer must lie, therefore, in the frequencies with which the various combinations of individual choices occur in the group setting. There are four such group states, $\mathrm{AAA}, \mathrm{AAB}, \mathrm{ABB}$, and $\mathrm{BBB}$, where each letter represents the terminal state in which one of the group members finds himself on the given trial. We assumed that the individuals make their decisions independently of others when in groups, and this assumption was tenable for the previous experiments on individual and 
TABLE 3

Predicted and Observed Proportions of Group Statis

\begin{tabular}{cccccc}
\hline & & \multicolumn{5}{c}{ Obtained } \\
\cline { 3 - 6 } $\begin{array}{c}\text { Group } \\
\text { state }\end{array}$ & Predicted & $\mathrm{I}-\mathrm{I}$ & $\mathrm{I}-\mathrm{M}$ & $\mathrm{I}-\mathrm{U}$ & $\mathrm{I}-\mathrm{MQ}$ \\
\hline AAA & .20 & .26 & .27 & .49 & .58 \\
$\mathrm{AAB}$ & .43 & .41 & .34 & .10 & .09 \\
$\mathrm{ABB}$ & .30 & .26 & .26 & .07 & .08 \\
$\mathrm{BBB}$ & .07 & .06 & .12 & .35 & .25 \\
\hline
\end{tabular}

group risk-taking and for the present I-M treatment, and even if it was not tenable, the predictions made under this assumption were not far from the observed results. But it is clearly not tenable for the I-U and I-MQ treatments. Table 3 shows the proportion of trials on which the four group states occurred in the four treatments and their predicted proportions when independence of choices is assumed and the choices are predicted by the terminal probabilities given by the parameter-free model.

It can be seen from Table 3 that there are indeed striking departures from the expected probabilities in the I- $U$ and I-MQ treatments. These departures are in the direction of increased occurrence of unanimities. The model predicts that there would be $27 \%$ unanimities, $20 \%$ favoring the frequent and $7 \%$ favoring the infrequent alternative. In the I-M treatment there is a tendency for these unanimities to be somewhat larger. However, in the I-U and I-MQ treatments these unanimities occur with an overwhelming frcquency. In the $\mathrm{I}-\mathrm{U}$ groups we have $84 \%$ and in I-MQ we have $83 \%$ unanimities, which are far above the expected percentages. It is also seen from Table 3 that the unanimities in the I-U groups favor the risky allernative more than they do in the I-MQ groups. It is therefore in this manner that the groups can maintain a level of risk-taking which the individual members consider acceptable, and which do not require the individuals to drastically change their own choice preferences.

\section{DISCUSSION}

As in the previous study on risk-taking in a two-choice situation (Zajonc et al., 1968) in which the probabilities of the two alternatives were .6 and .4 , the present experiment demonstrated a shift toward conservatism. But the significance of this shift is somewhat ambiguous because the I-I subjects also decreased their level of risk-taking during the second 180 trials. The parameter-free model was found again to 
predict individual risk-taking fairly reasonably. Also successful was the prediction to group risk-taking when the groups operated under the majority rule. The model predicted a conservative shift for I-M groups and a conservative shift did occur that was numerically not far removed from the predicted value. But, the data from the I-U and I-MQ treatments bore no resemblance to the predicted values.

Of course, it would be quite surprising if the parameter-free model did make accurate predictions for the I-U and the I-MQ cases. It would not be expected that individuals who settled on about $40 \%$ of risk-taking would accept $7 \%$ when they join a group working under the unanimity principle, or $80 \%$ when they work under minimal quorum rule. In fact, one of the purposes of introducing these treatments was to discover departures from the parameter-free model which would allow us to search for useful parameters.

It was initially expected that these parameters would pertain to the individual choice behavior, because that is where the main changes were anticipated. But we note that there were no dramatic changes in individual choice preferences when these choices were made in groups. While there was indeed some decline in individual choice preferences for the risky alternative in the three group treatments, this decline was not significant in groups reaching decisions by majority rule, and it was no larger than among subjects continuing to make decisions individually in I-U and I-MQ treatments.

The data then offer an indication that the individual members are quite resistant to changing their choice preferences. And when the individual maintains his choice preference the majority scheme leaves him with group choices that are not far removed from his own. He chooses the risky alternative $40.7 \%$ of the time when working alone, and a strict majority rule, with group members choosing independently of each other, predicts $36.3 \%$ risky choices made by the group. This represents less than a $5 \%$ shift, a change which is evidently quite tolerable. There must be a range of risk-taking which the person finds acceptable, whether he works alone or in groups. If he wants to remain within this range of risky choices when in a group which reaches decisions by unanimity, he must adjust his voting behavior. He can no longer predict his A's and B's as previously because other things held constant, the probability of a three-man group reaching a unanimous decision that favors the risky alternative is $.4^{3}$, which is very low indeed. ${ }^{3}$

\footnotetext{
${ }^{3}$ The majority scheme causes a minimal departure from the average of individual preference only when the group is quite small. For the present probabilities, .67 and .33 , a group of seven would vote by majority for the rare alternative only $30 \%$ of the time, and a group ten times as large would be extremely unlikely to vote for it at all.
} 
It appears from the results that not only does the individual maintain his own choice preference within a narrow range when in a group, but somehow his group generates joint decisions which also lie within the range of risk acceptable to him. Table 3 reveals how this consequence is achieved. It is significant that the sums of the AAA and AAB states are fairly similar across the various treatments. The sum of the proportions of these two states as predicted by the parameter-free model is .63. The proportions obtained for the I-M, I-U, and I-MQ treatments were .61, .59 , and .67. Of course, in the I-M treatment this sum consists of .27 for the AAA state and .34 for the $\mathrm{AAB}$ state, while we have .49 for AAA and .10 for $\mathrm{AAB}$ in the I-U groups, and .58 for $\mathrm{AAA}$ and .09 for $\mathrm{AAB}$ in the I-MQ groups. Similar effects obtain for the sums of the BBB and $\mathrm{ABB}$ states. It would appear, therefore, that in groups operating under unanimity and under the minimal quorum, when there are two choices for a given alternative, the probability of the third choice favoring that alternative is enhanced.

Let us first look at the I-U treatment. For any subject who has not yet made a decision, the probability that the two remaining group members have both chosen the infrequent alternative is, according to the predicted terminal probability, $.407^{2}=.17$. His own probability of being in state B is .407. Simply suppose that he is the last to vote one third of the time. Suppose further that when last and when the other two members have both voted B he will always vote B. Since he doesn't wish to abandon his own individual choice preference, which according to the model is .407 , he will be able to contribute only $1 / 3$ of his B-votes, because he is third only $1 / 3$ of the time. We have $.17+1 / 3(.407)=.31$. The data show a value .04 higher.

It should be noted at this point that as in the I-U treatment, there is pressure toward unanimity in the I-MQ treatment as well. Here the group choice is for B unless all vote for A. In these groups it is the likelihood of the conservative choice which would be depressed to about $.2 \mathrm{had}$ all three members retained their independence and their initial choice preferences. If this level of conservatism is too low, or putting it otherwise, if the resulting level of group risk is beyond the acceptable range, there will be pressure on the individual who hasn't yet voted and who is confronted by two $\mathrm{A}$ votes to vote for $\mathrm{A}$ also. That such pressures toward unanimity exist is clearly evident from Table 3. The I-U treatment has the greatest proportion of $\mathrm{BBB}$ states and the I-MQ treatment has the largest proportion of AAA states.

Let us then proceed with the I-MQ treatment in the same way as we did in the case of the I-U treatment. The likelihood of two members voting $\mathrm{A}$ when the third has not yet made up his mind is $.593^{2}=.35$. Adding one third of the individual's own A votes, or $1 / 3$ (.593) we have 
.55. This would be the predicted incidence of AAA states. The observed proportion of these states in the I-MQ treatment is not far removed, .58. The proportion of $B$ group choices in the I-MQ treatment is strictly dependent on the proportion of AAA group states, because, according to the minimal quorum rule, the group votes $B$ when at least one of its members votes for $\mathrm{B}$. The predicted proportion of $\mathrm{B}$ group choices for the I-MQ treatment is thus $1-.55=.45$, which exceeds the data by .03 .

It is of interest to note from Table 3 that the tendency toward unanimity generalizes to the irrelevant alternative as well. In the I-U treatment it is quite immaterial whether there are many or few AAA occurrences in the group. Yet the data show that in this treatment the preponderance of AAA states exceeded the expected value by a considerable margin. The same is true of the BBB states in the I-MQ treatment. Here it is also inconsequential whether there are many or few BBB statcs. Yet the proportion of these states in the I-MQ groups was .25, which exceeds chance expectations by 18 .

To summarize then, the individual who finds himself a part of a joint decision team does not seem to be ready to abandon his idiosyncratic preferences. Nor is he ready to allow the group of which he is a member to make decisions which would deviate significantly from his own preferences. This seems to be the case where this is quite difticult as, for instance, in groups that operate under the unanimity rule. The individual solves his problems by a judicious distribution of his choices over trials. All members of the group gauge their behavior so as to maximize unanimity when unanimity is the criterion of the group choice. We noted that in the I-U and the I-MQ treatments there were $84 \%$ and $83 \%$ of trials with unanimous choices. The remaining trials are apparently left for individuals to recapture their own idiosyncratic preferences. Thus the person gives up very little by joining the group that works under unanimity or the minimal quorum: he attempts to maintain his previous choice preference and he only abandons the freedom of selecting the trials on which he votes for A or B. Of course, in groups operating under majority rule he needn't abandon either his idiosyncratic choice preferences or the sequential pattern of these choices over trials. One should not mistake these pressures toward unanimity for conformity. It is quite clear that the individual does not give up the independence of his choices-only the timing of these choices. Wallach and Mabli (1970), using the standard choice dilemmas, also found little evidence of conformity effects in group risk taking. It should be noted, however, that other studies do indicate some tendencies toward uniformity or conformity, as there usually is a restriction of variance when individuals move from the solitary to the group condition. 
Because the majority rule affords the person not only the freedom to retain his idiosyncratic choice preferences but also the freedom to select the occasions on which he will exercise one preference or another, it would seem that a group free to determine its own decision scheme would lock in on the ma;ority process. In a recent study on binary choices in groups, Davis, Hornik, and Hornseth (1970), have found that while the majority scheme is indeed among the preferred ones, groups they observed are sufficiently flexible so as to allow themselves to function on some occasions by other schemes as well.

Because a different decision task than that conventionally used in risky shift studies was examined in the present experiment, one should be cautious in attempting to generalize to group processes occurring when individuals work with choice dilemmas. Nonetheless, on the basis of these results there is also cause to doubt whether risky shifts (or conservative shifts) obtained with conventional materials can be explained entirely in terms of changes in individual risk preferences with rules whereby these individual preferences are combined into a joint choice totally ignored.

\section{REFERENCES}

Bateson, N. Familiarization, group discussion, and risk taking. Journal of Experimental Social Psychology, 1966, 2, 119-129.

Brown, R. Social Psychology. New York: The Free Press, 1965.

Clausen, G. S. T. Risk taking in small groups. Unpublished doctoral dissertation. University of Michigan, 1965.

Davis, J. H., Honnik, J. A., \& Hornseth, J. P. Group decision schemes and strategy preferences in a sequential response task. Journal of Personality and Social Psychology, 1970, 15, 397-408.

Flanders, J. P., \& Thistlethwaite, D. L. Effects of familiarization and group discussion upon risk taking. Journal of Personality and Social Psychology, 1967, 5, 91-97.

SMoke, W., \& ZAJonc, R. B. On the reliability of group judgments and decisions. In J. Criswell, H. Solomon, and P. Suppes (Eds.) Mathematical methods in small group processes. Stanford, California: Stanford University Press, 1962.

STONER, J. A. F'. Risky and cautious shifts in group decisions: The influence of widely held values. Journal of Experimental Social Psychology, 1968, 5, 442459.

WALLACH, M. A., \& KOGAN; N. The roles of information, discussion, and consensus in group risk taking. Journal of Experimental Social Psychology, 1965, 1, 1-19.

Wallach, M. A., \& MaBLI, J. Information versus conformity in the effects of group discussion on risk taking. Joumal of Personality and Social Psychology, 1970, 14, $149-156$.

Wallach, M. A., Kogan, N., \& Bem, D. J. Diffusion of responsibility and level of risk taking in groups. Journal of Abnormal and Social Psychology, 1964, 68, 263-274.

Wallaci, M. A., Kogan, N., \& BuRt, R. B. Can group members recognize the 
effects of group discussion upon risk taking? Joumal of Experimental Social Psychology, 1965, 1, 379-395.

WINER, B. J. Statistical principles in experimental design. New York: McGraw-Hill, 1962.

Zajonc, R. B., Wolosin, R. J., Wolosin, M. A., \& Sherman, S. J. Individual and group risk-taking in a two-choice situation. Journal of Experimental Social Psychology, 1968, 4, 89-106.

Zajonc, R. B., Wolosin, R. J., Wolosin, M. A., \& Sherman, S. J. Group risktaking in a two-choice situation: Replication, extension, and a model. Journal of Experimental Social Psychology, 1969, 5, 127-140.

(Received June 17, 1970) 